\title{
A NOTE ON COWAN'S PROCESS OF SPACE DIVISION
}

\author{
J. MECKE, ${ }^{*}$ University of Jena
}

\begin{abstract}
Relations of a Markov chain describing the repeated splitting of polygons to processes known from queueing theory are pointed out.
\end{abstract}

R. Cowan presents an interesting interated random division procedure for polygons in his letter on pp. 233-234, which is related to the paper of Cowan and Morris (1988). His last model leads to a sequence of random polygons $P_{0} \supset P_{1} \supset P_{2} \supset \cdots$ the number of sides of which are denoted by $X_{0}, X_{1}, X_{2}, \cdots$ respectively. The random variables $Y_{n}=X_{n}-3$ $(n=0,1,2, \cdots)$ form a homogeneous Markov chain with state space $\{0,1,2, \cdots)$ and transition probabilities $\operatorname{pr}\left(Y_{n+1}=s \mid Y_{n}=r\right)=1 /(r+2)$ if $0 \leq s \leq r+1, \quad 0$ otherwise. The Poisson distribution with mean 1 is an equilibrium distribution for the Markov chain $Y_{0}, Y_{1}, Y_{2}, \cdots$. This fact is derived by direct computation.

The aim of this letter is to represent the process $Y_{0}, Y_{1}, Y_{2}, \cdots$ as an imbedded Markov chain with respect to a special birth and death process, for which it is well known that the Poisson distribution with mean 1 is an equilibrium distribution.

There is an analogue of the Cowan-Morris result in queueing theory. Let $Z(t)$ be the number of customers at time $t$ in a service system with an infinite number of servers, Poisson input of intensity $\lambda$, exponentially distributed service times with mean $1 / \lambda$ and assumptions of maximal independence (an $M / M / \infty$ system). Then the equilibrium distribution for the $Z$ process is the Poisson distribution with mean 1, cf. for example the Erlang formulas for service systems in Khinchin (1960). Now let $T_{1}, T_{2}, \cdots$ be the instants where customers arrive. Then the sequence $Z_{1}=Z\left(T_{1}-0\right), Z_{2}=Z\left(T_{2}-0\right), \cdots$ forms a homogeneous Markov chain, which has clearly the same equilibrium distribution as the process $Z(t)$. It can be shown that the transition probabilities of the Markov chains $Z_{1}, Z_{2}, \cdots$ and $Y_{1}, Y_{2}, \cdots$ coincide.

The mathematical essence of this model is the following. We consider a homogeneous birth and death process $Z(t)$ on the time half-axis $[0, \infty)$ with state space $\{0,1,2, \cdots\}$, constant birth rate $\lambda_{n}=\lambda>0$ and death rate $\mu_{n}=n \lambda ; n=0,1, \cdots$ If $T_{1}, T_{2}, \cdots$ are the instants where birth occurs, then the sequence of random variables $Z_{1}=Z\left(T_{1}-0\right), Z_{2}=Z\left(T_{2}-\right.$ $0), \cdots$ forms a homogeneous Markov chain with the same transition probabilities as the chain $Y_{1}, Y_{2}, \cdots$.

Having this in mind, we may think of the polygon splitting process as generated by a birth and death process in the following way. Consider the process $C_{0}, C_{1}, C_{2}, \cdots$ of corners of the polygons $P_{0} \supset P_{1} \supset P_{2} \supset \cdots$. Suppose $P_{n}$ has $C_{n}$ corners, numbered counterclockwise from a random reference corner as $1,2, \cdots, C_{n}$. The polygon $P_{n+1}$ may be viewed as the result of choosing a point on side $C_{n} \rightarrow 1$, and cutting from there to a point on side $k \rightarrow k+1$, where $k$ is chosen at random from $\left\{1,2, \cdots, C_{n}-1\right\}$, and taking $P_{n+1}$ as the polygon containing the corner 1 . Then $P_{n+1}$ has $k+2$ corners. The stochastic behaviour of the $C$ process is just as if the surplus corners $C-3$ each died at rate $\lambda$ and new corners are introduced at rate $\lambda$, with this process being sampled at instants of introduction of new corners.

\section{Acknowledgement}

I thank the referee for the suggestion presented in the last paragraph of this letter.

Received 23 September 1988; revision received 24 November 1988.

* Postal address: Department of Mathematics, The University, UHH, Jena, DDR-6900, GDR. 


\section{References}

Cowan, R. AND Morris, V. B. (1988) Division rules for polygonal cells. J. theor. Biol. 131, 33-42.

Cowan, R. (1989) The division of space and the Poisson distribution. Adv. Appl. Prob. 21, 233-234.

Khinchin, A. Y. (1960) Mathematical Methods in the Theory of Queueing. Griffin, London. 